runners), implying a decreased efficacy in jumpers and increased in runners.

Conclusions Throughout season neuromuscular adaptations occurred accordingly with sports gestures and this might explain the different results found between groups. Fatigue can be an important factor to explain the decrease of performance of runners. Sports with consecutive jumps maybe are related with the reduction of asymmetry, what does not happen between runners.

\section{INJURY BURDEN AND CHARACTERISTICS IN AESTHETIC SPORTS AMONG HIGH SCHOOL ADOLESCENTS}

1,2,3 Sheila Downie, ${ }^{1}$ Amanda M Black, ${ }^{1,2,4}$ Paul H Eliason, ${ }^{1,2,3,4,5,6}$ Carolyn Emery, ${ }^{1,2,3}$ Sarah J Kenny. 'Sport Injury Prevention Research Centre, Faculty of Kinesiology, University of Calgary, Calgary, Canada; ${ }^{2}$ Alberta Children's Hospital Research Institute, Cumming School of Medicine, University of Calgary, Calgary, Canada; ${ }^{3} O^{\prime} B r i e n$ Institute for Public Health, Cumming School of Medicine, University of Calgary, Calgary, Canada; ${ }^{4}$ Hotchkiss Brain Institute, Cumming School of Medicine, University of Calgary, Calgary, Canada; ${ }^{5}$ Community Health Sciences, Cumming School of Medicine, University of Calgary, Calgary, Canada; ${ }^{6}$ Department of Pediatrics, Cumming School of Medicine, University of Calgary, Calgary, Canada

\subsection{6/bjsports-2021-IOC.278}

Background Aesthetic sports require athletes to showcase extreme flexibility, aerial maneuvers and perform hard surface landings that may increase injury risk. However, very few studies have examined injury risk in this population.

Objectives To identify aesthetic sport injury prevalence among high school students and to describe the type, location and severity of injury in adolescents who practice aesthetic sports.

Design Cross-sectional study.

Setting High schools in Calgary area, Canada.

Participants Adolescent students $(\mathrm{n}=2029 ; 958$ male, 1048 female, 23 identified 'other'; ages 14-19 year) from 24 high schools.

Assessment of Risk Factors Self-reported participation in aesthetic sport (i.e., gymnastics, dance, figure skating) in previous one-year (based on top three sports for participation).

Main Outcome Measurements Self-reported injury (time loss/ medical attention), type, anatomical location, and severity. Proportions $[95 \%$ confidence intervals (CI)] were adjusted for cluster by school.

Results Among the 2029 students who completed the question about sport participation, 15\% (302/2029) participated in aesthetic sports (282 female, 20 males; dance (247/302; 82\%), gymnastics $(50 / 302 ; 16 \%)$, figure skating $(22 / 302 ; 7 \%)$. In the previous one-year, 74 females $(26.2 \%$; 95\% CI, 20.8-32.6) and 2 males (10.0\%; 95\% CI, 2.6-31.2) listed aesthetic sport injury as the most severe. Ankle (26.3\%; 95\% CI, 17.5-37.6), knee $(25.0 \%$; 95\% CI, 16.4-36.2), and back (9.2\%; 95\% CI 4.4-18.4) were the most common injury sites. Ligament sprains $(22.7 \%$; 95\% CI 14.4-33.7), muscle strains $(14.7 \%$; 95\%CI 8.2-24.9), and fractured bones $(12.0 \%$; 95\% CI 6.321.8) were most common injury types. Medical attention injury rate was 20.5/100 athletes/year and time-loss $>7$ days injury rate was 11.9/100 athletes/year.

Conclusions Aesthetic sport participation and injury rates among high school students are high. The most serious injuries reported were lower extremity injuries with a greater proportion of females reporting aesthetic sport injuries than males. Future research should focus on mitigation of lower extremity injuries among these high-risk aesthetic athletes.

\section{PREVALENCE AND BURDEN OF HEALTH PROBLEMS IN COMPETITIVE ADOLESCENT DISTANCE RUNNERS: A 6- MONTH PROSPECTIVE COHORT STUDY}

${ }^{1}$ Robert Mann, 2,3 Benjamin Clarsen, ${ }^{4}$ Carly McKay, ${ }^{4}$ Bryan Clift, ${ }^{1}$ Craig Williams, ${ }^{1}$ Alan Barker. 'Children's Health and Exercise Research Centre, University of Exeter, Exeter, UK; ${ }^{2}$ Department of Health Promotion, Norwegian Institute of Public Health, Oslo, Norway; ${ }^{3}$ Department of Sports Medicine, Norwegian School of Sports Sciences, Oslo, Norway; ${ }^{4}$ Department for Health, University of Bath, Bath, UK

\subsection{6/bjsports-2021-IOC.279}

Background Little is known about the overall health of adolescent distance runners.

Objective To describe all health problems (injuries and illnesses) in relation to type, location, incidence, prevalence, time loss, severity, and burden, in competitive adolescent distance runners in England.

Design Prospective observational study monitoring all health problems for 24-weeks between May and October (2019).

Setting Competitive adolescent distance runners (i.e., $800 \mathrm{~m}$ to $10,000 \mathrm{~m}$, including steeplechase) in England.

Patients (Or Participants) Distance runners (13-18 y) were invited to participate if they had achieved a top-50 performance in their age-group (U20, U17 and U15) during 2018. A total of 644 athletes were invited to take part, with 136 athletes (73 females) having enrolled and completed the study (lost to follow-up: $\mathrm{n}=7$ ).

Main Outcome Measurements The prevalence and burden of health problems was recorded using the Oslo Sports Trauma Research Center Questionnaire on Health Problems (OSTRC$\mathrm{H})$. The OSTRC-H was completed online, via Qualtrics, on a weekly basis.

Results A total of 363 health problems were registered during this study, including 213 injuries and 150 illnesses. At any time, 24\% [95\% Confidence Intervals (CI): 21-26\%] of athletes reported a health problem, with 11\% [95\% CI: 9-12\%] having experienced a health problem that had substantial negative impact on training and performance. Female athletes reported noticeably more illnesses, compared to male athletes, including higher prevalence, incidence, time loss, and severity. The most burdensome health problems, irrespective of sex, included lower leg, knee, and foot/toes injuries, alongside upper respiratory illnesses. The mean weekly prevalence of time loss was relatively low, regardless of health problem type or sex.

Conclusions Competitive adolescent distance runners are likely to be training and competing whilst concurrently experiencing health problems. These findings will support the development of injury and illness prevention measures.

\section{DOES THE FIFA 11+ SHOULDER INJURY PREVENTION PROGRAM REDUCE THE INCIDENCE OF UPPER EXTREMITY INJURIES AMONG SOCCER GOALKEEPERS? A RANDOMISED CONTROLLED TRIAL}

${ }^{1}$ Wesam Saleh A Al Attar, ${ }^{1,2}$ Mansour Abdullah Alshehri. 'Umm Al Qura University, Mecca, Saudi Arabia; ${ }^{2}$ University of Queensland, Brisbane, Australia

\section{$10.1136 /$ bjsports-2021-IOC.280}

Background Goalkeepers appear to be the players who are at most risk of sustaining a shoulder injury. Many researchers have reported that goalkeepers are more likely, than outfield players, to injure their upper limb. The FIFA $11+\mathrm{S}$ is 